\title{
Astigmatically compensated, high gain cooperative upconversion laser
}

\author{
P. Xie and S. C. Rand \\ Division of Applied Physics, Department of Electrical Engineering and Computer Science, University \\ of Michigan, 1301 Beal Avenue, Ann Arbor, Michigan 48109-2122
}

(Received 2 March 1992; accepted for publication 24 April 1992)

We demonstrate a cw Er:LiYF 4 trio laser in a 3-mirror cavity configuration, and investigate details of the mechanism yielding stimulated upconversion well above liquid nitrogen temperatures with an efficiency of $20 \%$.

In recent years a variety of schemes have been explored for compact, short-wavelength laser sources in solids. These include wide gap semiconductor diode lasers, ${ }^{1}$ nonlinear generation of optical harmonics by phase matching, ${ }^{2}$ quasiphase matching, ${ }^{3}$ and upconversion lasers in bulk media ${ }^{4}$ and fibers. ${ }^{5}$ In practical terms, while all these approaches have shown promise, all currently exhibit important limitations barring practical applications. Wide gap semiconductors present growth and doping problems and to date have not attained room-temperature operation by current injection. Nonlinear conversion techniques require critical alignment in bulk crystals and suffer reduced efficiencies in fibers and slab waveguides. Upconversion lasers operate by virtue of complex internal dynamics in which the basic mechanisms responsible for the upgrading of photon energy are still the subject of intense inquiry. Hence there are many questions, both practical and fundamental, which remain to be answered before limitations of the various approaches becomes fully evident.

In past upconversion laser research, low efficiencies were generally reported and liquid helium cryogenics were typically required. However many different mechanisms of upconversion exist and high efficiency, high-temperature cw operation is undoubtedly achievable. Here we report characteristics of an Er:LiYF 4 cooperative upconversion laser which attains $20 \%$ efficiency and operates at temperatures as high as $200 \mathrm{~K}$ in an open cavity configuration. Upconversion output at nearly twice the pump photon energy is achieved in our device uniquely through cooperative upconversion, one of three known types of upconversion which may be broadly categorized by their reliance on multiphoton, ${ }^{6}$ avalanche, ${ }^{7}$ or cooperative ${ }^{8}$ processes. The laser inversion itself is shown in the present case to be due entirely to a cooperative energy sharing process involving three atoms, very similar in nature to the monolithic Er$: \mathrm{CaF}_{2}$ trio laser we reported previously. ${ }^{9}$ By introducing a 3-mirror, astigmatically compensated cavity with Er: $\mathrm{LiYF}_{4}$ as the gain medium however, we have been able to study cavity losses, make precise assignments of excitation and emission wavelengths and investigate details of our nonlinear dynamics model in a fashion not possible in the original trio laser. In particular we show that $\mathrm{cw}$ operation occurs even when a single Stark level of the ${ }^{4} I_{13 / 2}$ state of $\mathrm{Er}^{3+}$ is simultaneously the pumped level and the terminal laser level. Pumping of the lowest Stark level of the ${ }^{4} I_{13 / 2}$ state, in which the predominant trio interaction takes place, also results in $\mathrm{cw}$ laser action. This substantiates the simple model and analysis of cooperative dynamics presented in our earlier paper.

The experimental setup is indicated in Fig. 1. A continuous-wave $\mathrm{NaCl}$ color center laser which was tunable in the region of $1.55 \mu \mathrm{m}$ was used to provide resonant excitation of the ${ }^{4} I_{13 / 2}$ level of trivalent erbium, the laser active species. The gain medium consisted of a $3 \mathrm{~mm}$ thick crystal of $5 \%$ Er:LiYF ${ }_{4}$ oriented at Brewster's angle $\left(\theta_{\mathrm{B}}\right.$ $=55.5^{\circ}$ ) with its optic axis in the plane of incidence parallel to the crystal surface. This orientation permits gain extraction on either $\pi$ or $\sigma$ polarized transitions. The laser crystal was mounted in vacuum on the cold finger of a liquid helium open cycle cryostat capable of operation down to $6 \mathrm{~K}$. Using a value for the extraordinary refractive index ${ }^{10}$ of $n_{e}(\theta)=1.453$, an interarm angle of $\theta=26.2^{\circ}$ was calculated for compensation of the astigmatism at the trio laser wavelength. To minimize internal losses while retaining experimental flexibility, all focusing and cavity optics except the output coupler were placed inside the dewar housing. External feedthroughs were used to permit cavity adjustments in vacuum, and independent $X Y Z$ positioning of the crystal was made possible by flexible bellows connecting the dewar head to the vacuum chamber.

To verify that inversion in this system arises strictly from energy-sharing interactions of trios of $\mathrm{Er}$ ions initially excited to the ${ }^{4} I_{13 / 2}$ level, we measured the time and power dependencies of upconversion emission at two wavelengths, as shown in Figs. 2(a) and 2(b) respectively. The Fig. 2(b) inset indicates the dynamics schematically. The time-domain measurements revealed the evolution of upconversion population in the ${ }^{4} S_{3 / 2}$ and ${ }^{4} I_{11 / 2}$ levels following impulse excitation of the ${ }^{4} I_{13 / 2}$ level. They were made with an $S-1$ photomultiplier terminated in $50 \mathrm{ohms}$ and amplified with a transimpedance amplifier of DC-13 $\mathrm{MHz}$ bandwidth. Results in Fig. 2(a) clearly show that no population is observed in either the ${ }^{4} I_{11 / 2}$ or the ${ }^{4} S_{3 / 2}$ state during the excitation pulse of $100 \mu$ s duration. Upconversion emission grows and peaks on a timescale much longer than the pulsewidth. This is far too long for any process involving the absorption of more than one photon from the incident pulse by a single atom, yet Fig. 2(b) indicates that upconversion fluorescence intensity from the ${ }^{4} S_{3 / 2}$ level varies as the incident intensity cubed, when saturation of the pump transition is carefully avoided.

These observations are similar to those ${ }^{9}$ in $\mathrm{CaF}_{2}$, except that the small prompt fluorescence signal observed in the earlier work is not observed at all in $\mathrm{LiYF}_{4}$. Therefore all multiphoton excitation processes are ruled out by the 


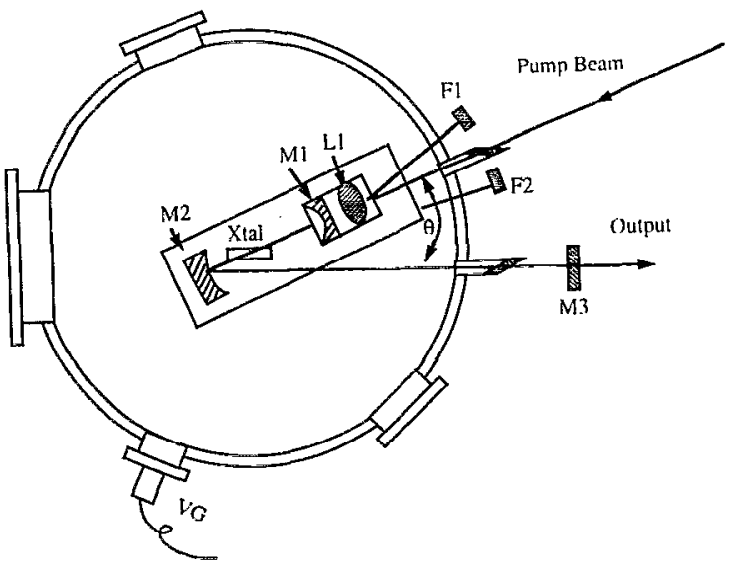

FIG. 1. Experimental setup. The laser crystal was suspended on a cold finger in a vacuum chamber containing focusing lens $(L 1)$, cavity mirrors ( $M 1$ and $M 2$ ), vacuum gauge (VG), and mechanical feedthroughs ( $F 1$ and $F 2$ ). Output coupler $M 3$ was located outside the dewar, beyond a fused silica Brewster window.

time dependence of fluorescence excitation shown in Fig. 2 (a) for the power levels of this experiment, and the trio process involving promotion of one $\mathrm{Er}^{3+}$ ion to the ${ }^{2} H_{11 / 2}$ manifold at the expense of de-excitation of two neighbors is
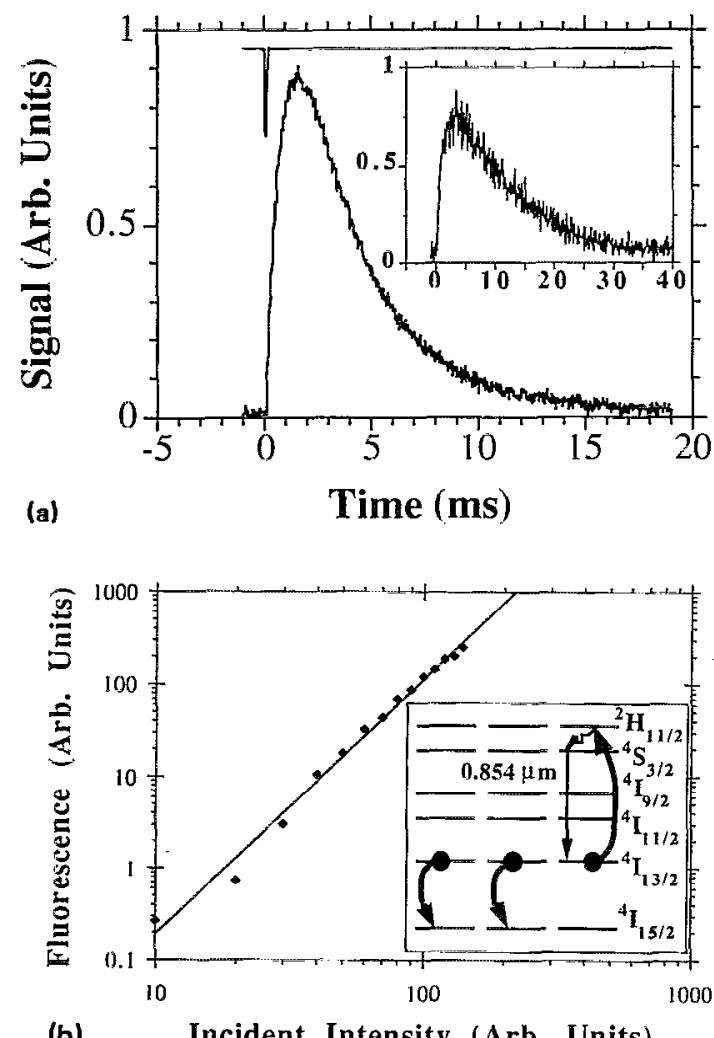

(b) Incident Intensity (Arb. Units)

FIG. 2. (a) Temporal development of $0.8506 \mu \mathrm{m}$ fluorescence intensity following excitation with a $100 \mu$ s pulse of peak intensity $2 \mathrm{~kW} / \mathrm{cm}^{2}$ (inverted trace). Inset: Evolution of pair-excited $0.98 \mu \mathrm{m}$ fluorescence from the ${ }^{4} I_{11 / 2}$ state under identical conditions. (b) Dependence of upconversion fluorescence intensity on incident intensity below $0.2 \mathrm{~W} / \mathrm{cm}^{2}$. Solid curve is a power law, with a best-fit slope of $2.8 \pm 0.1$. Inset: Schematic of the relevant trio process in erbium.

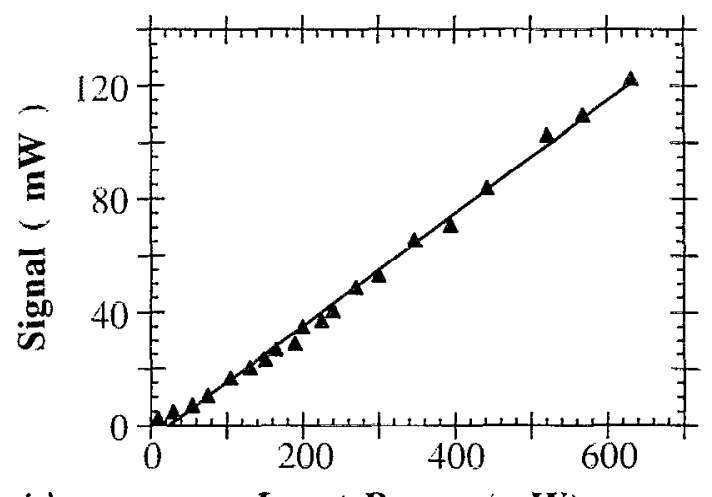

(a)

Input Power $(\mathrm{mW})$

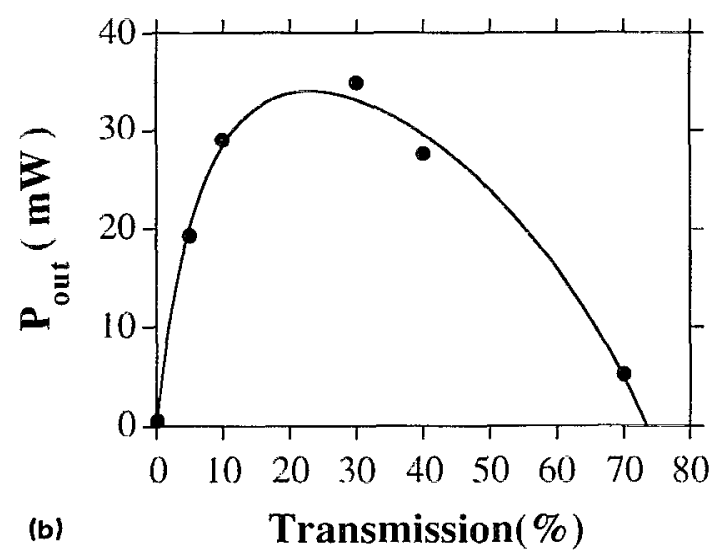

FIG. 3. (a) Laser output power $(0.8543 \mu \mathrm{m})$ vs input power at $77 \mathrm{~K}$. (b) Output power vs output reflectivity at a fixed pump power of $200 \mathrm{~mW}$ focused to a spot of radius $18 \mu \mathrm{m}$ in the gain medium.

uniquely established as the upconversion mechanism. The predominance of this cooperative interaction is quite different from behavior reported by other researchers who used excitation wavelengths shorter than that of the first $\mathrm{Er}^{3+}$ resonance. It is therefore important to recognize that upconversion dynamics strongly depend on the details of the initially prepared state. Ilere we have deliberately prepared the ensemble of Er ions in the lowest excited state to isolate the multiatom or cooperative upconversion process and study its capabilities alone.

Upconversion laser experiments were performed with cooled samples and a variety of output couplers ranging between $0.1 \%$ and $70 \%$ transmission. An example of output versus input power at liquid nitrogen temperature is given in Fig. 3(a) for output coupling of $20 \%$. For this coupling, a threshold of approximately $5 \mathrm{~mW}$ was observed, somewhat below the intercept in the figure, and slope efficiency was $20 \%$. Overall efficiency was $20 \%$ for a pump-limited maximum output power of $120 \mathrm{~mW}$. No evidence of laser output saturation was observed at these high power levels. Output power versus output coupler transmission is plotted in Fig. 3(b). The solid curve is a fit to the data of the standard expression for output power. ${ }^{11}$ Optimum output coupling was determined to be $23 \%$, with gain and internal cavity losses estimated to be $71 \%$ and $4.3 \%$ per pass, respectively. 


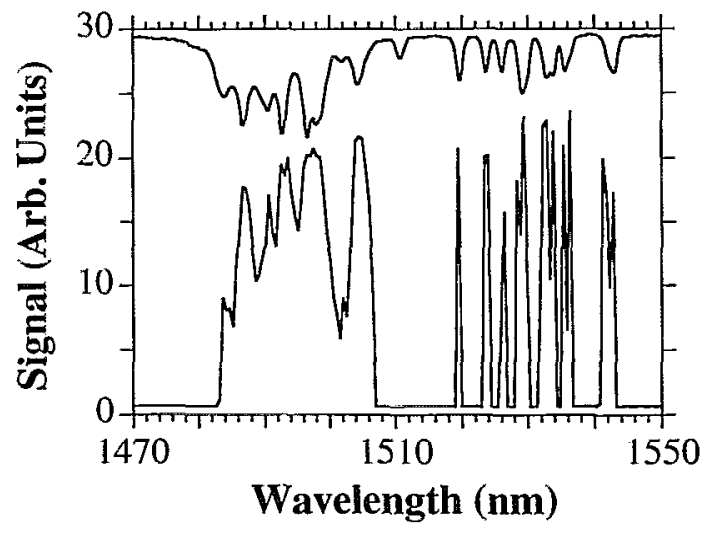

FIG. 4. Excitation spectrum of upconversion laser action in $\mathrm{Er}: \mathrm{LiYF}_{4}$ at $77 \mathrm{~K}$, and the Er absorption spectrum (inverted). The absorptive transition from the lowest ground state to the bottom of the first excited state manifold $\left[{ }^{4} I_{15 / 2}(1)-{ }^{4} I_{13 / 2}(1)\right]$ occurs at $\lambda=1.530 \mu \mathrm{m}$.

For excitation tuned to any Er absorption line in the range $1.45-1.51 \mu \mathrm{m}$, laser output occurred at wavelength at $0.8506 \mu \mathrm{m}$, corresponding to ${ }^{4} S_{3 / 2}(1)-{ }^{4} I_{13 / 2}(4)$ transition. This laser transition has mixed $\pi, \sigma$ character ${ }^{12}$ due to Kramers degeneracy and terminates in the fourth ${ }^{4} I_{13 / 2}$ Stark level $140 \mathrm{~cm}^{-1}$ above the bottom of the manifold. However lasing characteristics were unchanged even when this fourth Stark level was selected as the pumped level. This result is indicated in the laser excitation spectrum of Fig. 4. For pumping wavelengths at Er absorption lines longer than $1.5045 \mu \mathrm{m}$, the output wavelength shifted to $0.8543 \mu \mathrm{m}$ corresponding to the ${ }^{4} S_{3 / 2}(1)-{ }^{4} I_{13 / 2}(6)$ transition. Specification of Stark levels (in brackets) was not attempted in our earlier paper due to the high doping level and multiple-site character of $\mathrm{CaF}_{2}$. Problems related to spectroscopic assignments are overcome in $\mathrm{Er}: \mathrm{LiYF}_{4}$, since $\mathrm{Er}$ is found in a single tetragonally distorted site with partially polarized spectra which readily permit identification ${ }^{12}$ of all seven (Kramers degenerate) ${ }^{4} I_{15 / 2}(1)$ $-{ }^{4} I_{13 / 2}(n)$ Stark components at liquid helium temperatures. Of particular significance here is the observation that the lower laser level can be coincident with the pumped level, in the manifold which is simultaneously the origin of the trio interaction.

True cw operation was obtained under all pumping conditions of the Er:LiYF 4 trio laser. This contrasts recent observations of sustained oscillations at high levels of excitation in the pair-pumped $\mathrm{Er}: \mathrm{CaF}_{2}$ laser, which is also a cooperative laser. ${ }^{13}$ Superior stability of the trio laser may be related to the fact that the ${ }^{4} S_{3 / 2}$ upper state lifetime ${ }^{14}$ is much shorter than that of the ${ }^{4} I_{11 / 2}$ upper state of the pair laser. Our continuous-wave operation also contrasts sharply with self-pulsing observed on the green transition from the same upper state in Er:LiYF 4 when alternate excitation methods are used. ${ }^{6}$ Self $Q$-switching on the green transition has been attributed ${ }^{4}$ to excited state absorption (ESA) from the ${ }^{4} I_{13 / 2}$ state, self-absorption which may be absent at $0.85 \mu \mathrm{m}$. However, stability may also be imparted by the inherently sluggish response of the cooperative upconversion mechanism to changes in intracavity photon density, compared to the fast response time of multiphoton upconversion mechanisms. Experiments in progress on the $551 \mathrm{~nm}$ laser transition are expected to provide further insight on this point.

In summary, we have demonstrated cw cooperative upconversion laser operation in a new host with a cavity configuration amenable to the study of laser dynamics and the measurement of internal losses. With detailed optical spectroscopy we have confirmed that excitation mechanisms other than cooperative upconversion contribute negligibly to the inversion of this trio laser. True continuouswave operation can be sustained by the spontaneous trio interaction alone, even when the lower laser level is pumped directly. This approach permits surprisingly efficient operation to temperatures as high as $200 \mathrm{~K}$.

Research sponsored by the Air Force Office of Scientific Research (AFSC) under Grant AFOSR-91-0369.

'M. Haase, J. Qiu, J. DePuydt, and H. Cheng, Appl. Phys. Lett. 59, 1272 (1991); H. Jeon, J. Ding, W. Patterson, A. Nurmikko, W. Xie, D. Grillo, M. Kobayashi, and R. Gunshor, Appl. Phys. Lett. 59, 3619 (1991).

${ }^{2}$ L. Goldberg and M. K. Chun, Appl. Phys. Lett. 55, 218 (1989); U. Osterberg and W. Margulis, Opt. Lett. 12, 57 (1987).

${ }^{3}$ J. A. Armstrong, N. Bloembergen, J. Ducuing, and P. S. Pershan, Phys. Rev. 127, 1918 (1962); N. Bloembergen and A. J. Sievers, Appl. Phys. Lett. 17, 483 (1970); A. Feisst and P. Koidl, Appl. Phys. Lett. 47, 1125 (1985).

${ }^{4}$ L. F. Johnson and H. J. Guggenheim, Appl. Phys. Lett. 19, 44 (1971).

${ }^{5}$ J. Y. Allain, M. Monerie, and H. Poignant, Electron. Lett. 26, 168 (1989); J. Y. Allain, M. Monerie, and H. Poignant, Electron. Lett. 26, 262 (1990).

${ }^{6}$ A. J. Silversmith, W. Lenth, and R. M. MacFarlane, Appl. Phys. Lett. 51, 1977 (1987); R. M. MacFarlane, F. Tong, A. J. Silversmith, and W. Lenth, Appl. Phys. Lett. 52, 1300 (1988); D. C. Nguyen, G. E. Faulkner, and M. Dulick, Appl. Opt. 28, 3553 (1989); R. A. McFarlane, Appl. Phys. Lett. 54, 2301 (1989).

${ }^{7}$ M. E. Koch, A. W. Kueny, and W. E. Case, Appl. Phys. Lett. 56, 1083 (1990).

${ }^{8}$ P. Xie and S. C. Rand, Opt. Lett. 15, 848 (1990).

${ }^{9}$ P. Xie and S. C. Rand, Appl. Phys. Lett. 57, 1182 (1990).

${ }^{10}$ D. E. Castleberry and A. Linz, Appl. Opt. 14, 2056 (1975).

${ }^{11}$ A. E. Siegman, Lasers (University Science Books, Mill Valley, CA, 1986), Chap. 12.

${ }^{12}$ S. M. Kulpa, J. Phys. Chem. Solids 36, 1317 (1975).

${ }^{13} \mathrm{P}$. Xie and S. C. Rand, Conference on Lasers \& Electro-optics CLEO '92, Anaheim, California, May 10-15, 1992, paper CFE-4.

${ }^{14}$ S. A. Pollack, D. B. Chang, and M. Birnbaum, Appl. Phys. Lett. 54, 869 (1989). 